\title{
Neutron Scattering Investigation of the Spin Ice State in $\mathrm{Dy}_{2} \mathrm{Ti}_{2} \mathrm{O}_{7}$.
}

\author{
T. Fennell \\ The Royal Institution of Great Britain, 21 Albemarle Street, London, W1S 4BS, United Kingdom \\ O. A. Petrenkd $*$ and B. Fåł \\ ISIS Facility, Rutherford-Appleton Laboratory, Chilton, Didcot, OX11 0QX, United Kingdom
}

\author{
S. T. Bramwel围 \\ Department of Chemistry, University College London, \\ 20 Gordon Street, London, WC1H OAJ, United Kingdom \\ M. Enjalran\$ T. Yavors'kii, and M. J. P. Gingras \\ Department of Physics, University of Waterloo, Ontario, N2L 3G1, Canada \\ R. G. Melko \\ Department of Physics, University of California, Santa Barbara, CA 93106-9350, USA \\ G. Balakrishnan \\ Department of Physics, University of Warwick, Coventry, CV4 7AL, United Kingdom
}

(Dated: October 4, 2018)

\begin{abstract}
$\mathrm{Dy}_{2} \mathrm{Ti}_{2} \mathrm{O}_{7}$ has been advanced as an ideal spin ice material. We present a neutron scattering investigation of a single crystal sample of ${ }^{162} \mathrm{Dy}_{2} \mathrm{Ti}_{2} \mathrm{O}_{7}$. The scattering intensity has been mapped in zero applied field in the $h, h, l$ and $h, k, 0$ planes of reciprocal space at temperatures between $0.05 \mathrm{~K}$ and $20 \mathrm{~K}$. The measured diffuse scattering has been compared with that predicted by the dipolar spin ice model. The comparison is good, except at the Brillouin zone boundaries where extra scattering appears in the experimental data. It is concluded that the dipolar spin ice model provides a successful basis for understanding $\mathrm{Dy}_{2} \mathrm{Ti}_{2} \mathrm{O}_{7}$, but that there are issues which remain to be clarified.

PACS numbers: $75.50 \mathrm{Lk}, 75.40 \mathrm{Cx}, 75.25+\mathrm{z}$
\end{abstract}

\section{INTRODUCTION}

The concept of spin ice $\frac{1}{\underline{1}}$ has appeared recently as a result of a direct analogy between the statistical mechanics of O-H bonds in Pauling's model of water ice 2.3 and those of the localized magnetic moments of some highly frustrated magnets ${ }^{1,4.5}$. These materials, typified by $\mathrm{Ho}_{2} \mathrm{Ti}_{2} \mathrm{O}_{7} \frac{1}{1}, \mathrm{Dy}_{2} \mathrm{Ti}_{2} \mathrm{O}_{7} \stackrel{4}{\frac{4}{4}}$ and $\mathrm{Ho}_{2} \mathrm{Sn}_{2} \mathrm{O}_{7} \frac{6}{}$, provide almost ideal experimental realizations of spin ice, a magnetic "sixteen vertex" model7. Their temperature-field phase diagrams are exceedingly rich and have revealed several new magnetic phases and transitions $1,8,9,10,11$. The magnetic dynamics of the spin ices also show some unique properties including a crossover from Debye-like spin relaxation ${ }^{12,13,14}$ to quantum tunnelling dynamics ${ }^{15.16}$ at the unusually high temperature of $15 \mathrm{~K}$. The current paper builds on earlier work ${ }^{1.6 .17 .18}$ to characterize the neutron scattering of spin ice materials. In it, we report the first neutron scattering images of the spin ice state of $\mathrm{Dy}_{2} \mathrm{Ti}_{2} \mathrm{O}_{7} 19$ which are compared with theoretical predictions $20,21,22$.

In $\mathrm{Dy}_{2} \mathrm{Ti}_{2} \mathrm{O}_{7}$, as well as in the other spin ice materials $\mathrm{Ho}_{2} \mathrm{Ti}_{2} \mathrm{O}_{7}$ and $\mathrm{Ho}_{2} \mathrm{Sn}_{2} \mathrm{O}_{7}$, the rare earth moments ('spins') are arranged on a pyrochlore lattice of linked tetrahedra (see Fig. 1). Strong crystal field anisotropy ${ }^{23}$ confines each spin as an Ising-like doublet to the local trigonal axis that connects the center of the elementary tetrahedron to its vertex. With this constraint and ferromagnetic coupling, the magnetic ground state of a single tetrahedron is defined by a "two spins in, two spins out" rule, analogous to the Bernal-Fowler ice rule that controls the proton arrangements in water ice 24 . This rule minimizes only four of the six near neighbor magnetic interactions and so the system is highly frustrated, sharing with ice the property of zero point entropy 1.2.3.4. In the spin ice materials, the ferromagnetic near neighbor coupling arises from the strong dipole-dipole interaction between neighboring spins ${ }^{20}$. If the dipolar interaction is truncated at nearest neighbors one has the "near neighbor" spin ice model ${ }^{1.7}$ (note that dipole interactions are equivalent to exchange terms for Ising spins). This approximation captures the bulk properties of the spin ice materials $1,4,25,26,27$ and gives a qualitative description of the microscopic spin correlations, as determined by neutron scattering 1.17 .

The subject of this paper, $\mathrm{Dy}_{2} \mathrm{Ti}_{2} \mathrm{O}_{7}$, has previously been investigated by bulk methods 4.12 .27 .28 . In zero field, the specific heat resembles a Schottky anomaly, peaking at $1.24 \mathrm{~K}$ and falling to zero at lower temperatures. Unlike its $\mathrm{Ho}_{2} \mathrm{Ti}_{2} \mathrm{O}_{7}$ analogue, which shows an extra peak due to freezing of the nuclear spins ${ }^{17.29}, \mathrm{Dy}_{2} \mathrm{Ti}_{2} \mathrm{O}_{7}$ shows no other specific heat contributions down to 0.2 
FIG. 1: Top: a unit cell of the pyrochlore lattice which consists of four interpenetrating face centered cubic (fcc) Bravais sublattices. Two spins related by an fcc translation are shown with antiferromagnetic correlation. Correlations of this type give rise to the zone boundary scattering observed experimentally. Bottom: the spin ice state on a single tetrahedron (bottom). A macroscopic spin ice state is constructed by stacking such local arrangements on the lattice.

\begin{tabular}{|c|c|c|c|}
\hline Isotope & $\begin{array}{c}\text { Natural } \\
\text { Abundance } \\
(\%)\end{array}$ & $\begin{array}{c}\text { Sample } \\
\text { Content } \\
(\%)\end{array}$ & $\begin{array}{c}\sigma_{a} \\
(\text { barn })\end{array}$ \\
\hline \hline Natural & - & - & $994 .(13)$. \\
\hline${ }^{156} \mathrm{Dy}$ & 0.06 & $<0.01$ & $33 .(3)$. \\
\hline${ }^{158} \mathrm{Dy}$ & 0.1 & $<0.01$ & $43 .(6)$. \\
\hline${ }^{160} \mathrm{Dy}$ & 2.34 & 0.02 & $56 .(5)$. \\
\hline${ }^{161} \mathrm{Dy}$ & 19 & 0.47 & $600 .(25)$. \\
\hline${ }^{162} \mathrm{Dy}$ & 25.5 & 96.8 & $194 .(10)$. \\
\hline${ }^{163} \mathrm{Dy}$ & 24.9 & 2.21 & $124 .(7)$. \\
\hline${ }^{164} \mathrm{Dy}$ & 28.1 & 0.5 & $2840 .(40)$. \\
\hline Sample & - & - & 207.6 \\
\hline
\end{tabular}

TABLE I: Isotopic abundances and absorption cross sections $\left(\sigma_{a}\right)$ of natural dysprosium and the enriched sample used in these experiments 32 .

$\mathrm{K}$, where the specific heat becomes exponentially small. It has thus proved highly favorable for bulk measurements, having been used to estimate the zero point entropy in zero field ${ }^{4}$, and to demonstrate the existence of "kagomé ice", a field induced state that retains zero point entropy 9.28 . However, owing to its large neutron absorption, $\mathrm{Dy}_{2} \mathrm{Ti}_{2} \mathrm{O}_{7}$ has not yet been extensively studied by neutron scattering ${ }^{19.30}$, the technique that was crucial in establishing the existence of the spin ice state in $\mathrm{Ho}_{2} \mathrm{Ti}_{2} \mathrm{O}_{7} \frac{1.17}{1}$ and $\mathrm{Ho}_{2} \mathrm{Sn}_{2} \mathrm{O}_{7} \stackrel{6}{\text {. }}$.

To address the problem of large neutron absorption in natural dysprosium based materials we have grown an isotopically enriched crystal of ${ }^{162} \mathrm{Dy}_{2} \mathrm{Ti}_{2} \mathrm{O}_{7}$ specifically for use in neutron scattering experiments. The details of the sample and the experiments performed on it, are given in section $\llbracket \mathrm{A}$. The experiments were designed to examine the nature of the presumed spin ice state that is established when the sample is cooled in zero applied magnetic field ${ }^{4,20}$. The data were modelled using the "dipolar" spin ice model that considers further neighbor dipole-dipole interactions as well as a small antiferromagnetic exchange coupling that can be estimated by fitting the susceptibility ${ }^{31}$ or specific heat ${ }^{20}$. Details of the dipolar spin ice model ${ }^{20}$, as applied to $\mathrm{Dy}_{2} \mathrm{Ti}_{2} \mathrm{O}_{7}$, are given in section $\llbracket \mathrm{B}$ of the current paper, while the comparison of theory and experiment is given in section III The paper is concluded with a discussion of the results, section IV]

\section{EXPERIMENTAL}

\section{A. Neutron Scattering}

Neutron scattering measurements were carried out on PRISMA at the ISIS pulsed neutron source of the Rutherford-Appleton Laboratory. A pulsed white neutron beam is incident on the sample. PRISMA views a cold $(95 \mathrm{~K})$ methane moderator and a supermirror guide system, providing a high flux of long wavelength neutrons allowing measurement at relatively low $|\mathbf{Q}|$. The neutrons scattered from the sample are recorded in sixteen detectors which cover a range of $16^{\circ}$ in scattering angle. From the neutron time of flight, the scattering angle and the orientation of the sample with respect to the incoming beam, the scattered intensity can be determined as a function of the position in reciprocal space. By rotating the crystal about the vertical axis, intensity maps covering a large region of the scattering plane can be constructed. This method is particularly useful for studies of diffuse magnetic scattering. The average scattering angle $\phi$ of the sixteen detectors used for the measurements is chosen as a compromise between flux (at a given wavevector) and background (due to air scattering). The advantages of measuring the diffuse magnetic neutron scattering on PRISMA have been clearly demonstrated using the example of $\mathrm{Ho}_{2} \mathrm{Ti}_{2} \mathrm{O}_{7}$, where the dipolar spin ice nature of the zero field spin correlations has been unambiguously established 17 . In that and the current experiment the neutron scattering was measured in the static approximation ${ }^{33}$.

Natural dysprosium contains seven isotopes, several of which absorb neutrons quite strongly. This can make the detection of weaker effects like diffuse scattering difficult or impossible. To reduce this, an isotopically enriched sample was used. The natural abundances, scattering lengths and cross sections for natural dysprosium and our sample are given in Table प Using the ${ }^{162} \mathrm{Dy}$ isotope the average absorption cross-section $\bar{\sigma}_{a}$ is reduced by a factor of 4.8 compared to natural Dy. Since absorption attenuates scattered intensity by a factor of the form $\exp \left(-N \bar{\sigma}_{a} \lambda\right.$ ) (where $N$ is the number density of scatterers per unit volume, $\bar{\sigma}_{a}$ is the average absorption cross section, usually tabulated for $2200 \mathrm{~m} \mathrm{~s}^{-1}$ neutrons, and $\lambda$ is the neutron wavelength scaled to the value used in $\bar{\sigma}_{a}$ ) this makes a significant difference at long wavelengths.

A large high quality single crystal was grown using an infra-red double mirror image furnace 34 . The crystal has a diameter of $\approx 0.4 \mathrm{~cm}$, length $\approx 1.5 \mathrm{~cm}$, and is translucent amber-red in color. It has a cylindrical form typical of image furnace grown crystals. The crystal was varnished into a large copper support to ensure good thermal contact.

Using a ${ }^{3} \mathrm{He}$ sorption refrigerator insert in an Orange cryostat, the $h, h, l$ plane of reciprocal space was mapped in zero field at $20 \mathrm{~K}$ and $0.3 \mathrm{~K}$ using the average scattering angle $\phi=48^{\circ}$. The same plane was mapped at 0.3 $\mathrm{K}$ and $1.3 \mathrm{~K}$ using the average scattering angle $\phi=32^{\circ}$. 
Using a dilution refrigerator insert in an Oxford Instruments cryomagnet the $h, k, 0$ and $h, h, l$ planes of reciprocal space were mapped in zero field at $0.05 \mathrm{~K}$. These maps are shown in Fig. 2 All parts of the sample environment in the beam were made of aluminium (other than the copper support), which gives rise to powder lines at $|\mathbf{Q}|=2.70,3.12$ and $4.40 \AA^{-1}$.

Standard data reduction procedures were applied to transform time of flight and angle information to reciprocal space, to correct for absorbtion in the sample, and to normalize the data to an absorption corrected vanadium run. The latter corrects for the wavelength-dependent flux profile and detector efficiency. Despite the five-fold reduction in absorption cross section compared to natural dysprosium, the data analysis procedure revealed that absorption by the $\mathrm{Dy}_{2} \mathrm{Ti}_{2} \mathrm{O}_{7}$ crystal was still significant and needed to be taken into account. The absorption correction was made by calculating an attenuation coefficient $(A(s, \phi))$ by which the data could be divided. The coefficient for a cylinder of radius $R$ was originally derived by Sears 35 and is

$$
A(s, \phi)=\left[1+4 b s^{2}-0.5 s^{2} \cos ^{2}(\phi / 2)\right] \times \exp (-2 a s),
$$

where $a=(8 / 3) \pi, b=\left(1-a^{2}\right) / 2, s=\mu R$ and $\phi$ is the scattering angle. In this formula, $\mu$, the absorption coefficient, depends on both the scattering cross section and the wavelength dependent absorption cross section.

\section{B. Monte Carlo Simulations}

To model the experimental data for $\mathrm{Dy}_{2} \mathrm{Ti}_{2} \mathrm{O}_{7}$, we use a Hamiltonian appropriate for the $\langle 111\rangle$ Ising pyrochlores:

$$
\begin{aligned}
& H=-J \sum_{\langle(i, a),(j, b)\rangle} \boldsymbol{S}_{i}^{a} \cdot \boldsymbol{S}_{j}^{b} \\
& +D R_{\mathrm{nn}}^{3} \sum_{(i, a)>(j, b)}\left(\frac{\boldsymbol{S}_{i}^{a} \cdot \boldsymbol{S}_{j}^{b}}{\left|\boldsymbol{R}_{i j}^{a b}\right|^{3}}-\frac{3\left(\boldsymbol{S}_{i}^{a} \cdot \boldsymbol{R}_{i j}^{a b}\right)\left(\boldsymbol{S}_{j}^{b} \cdot \boldsymbol{R}_{i j}^{a b}\right)}{\left|\boldsymbol{R}_{i j}^{a b}\right|^{5}}\right),
\end{aligned}
$$

where $\boldsymbol{S}_{i}^{a}=\hat{z}^{a} \sigma_{i}^{a}$ represents an Ising moment of magnitude $\left|\boldsymbol{S}_{i}^{a}\right|=1$ at fcc lattice site $i$ and tetrahedral basis position $a$, with its quantization axis $\left(\hat{z}^{a}\right)$ oriented along the local trigonal axis (a member of the $\langle 111\rangle$ set), and $\sigma_{i}^{a}= \pm 1$. The nearest neighbor exchange energy is $J_{\mathrm{nn}}=J / 3$ and the nearest dipole-dipole strength is $D_{\text {nn }}=5 D / 3$, where $D=\left(\mu_{o} / 4 \pi\right) \mu^{2} / R_{\text {nn }}^{3}$ and $\mu$ is the moment of the $\mathrm{Dy}^{3+}$ ion. $\boldsymbol{R}_{i j}^{a b}$ is the vector separation between moments $\boldsymbol{S}_{i}^{a}$ and $\boldsymbol{S}_{j}^{b}$.

The model (2), with parameters appropriate to $\mathrm{Dy}_{2} \mathrm{Ti}_{2} \mathrm{O}_{7}$, orders magnetically at $0.18 \mathrm{~K}^{21}$. The real material does not show this ordering transition, but we test the hypothesis that the correlated paramagnetic state just above the transition temperature will provide an adequate description of the spin ice state. Equation 2 was simulated on a pyrochlore lattice of $4 \times 4 \times 4$ cubic cells $(N=1024$ spins $)$ with periodic boundary conditions. The long range nature of the dipole-dipole interactions was properly handled by using standard Ewald techniques for real space dipoles ${ }^{20}$. The model employed energy parameters appropriate for $\mathrm{Dy}_{2} \mathrm{Ti}_{2} \mathrm{O}_{7}, J=-3.72$ $\mathrm{K}$ and $D=1.41 \mathrm{~K}^{20}$.

At temperatures below $1 \mathrm{~K}$ or so the system is only able to access states within the spin ice manifold. Single spin flip dynamics require the introduction of ice rule defects. This means that the acceptance rate of single spin flips becomes extremely small and ability to equilibrate the system below $0.3 \mathrm{~K}$ becomes dubious ${ }^{20.21}$. In order to improve on approach to equilibrium, dynamics were restored by using a loop algorithm ${ }^{21}$. To simulate scattering data the system was placed in the ordered groundstate found by Melko et al $l^{21}$, at 0.4 K. $5 \times 10^{6}$ Monte Carlo steps (MCS) per spin and $5 \times 10^{6}$ loop attempts were made to equilibrate the system and bring it to a paramagnetic state. Loop attempts were therefore separated by $N=1024 \mathrm{MCS}$. The system was then cooled to $0.3 \mathrm{~K}$ where the same number of single spin flip and loop attempt steps were used for equilibration and then data collection (see Refs. 172036 for more details).

There are 12 symmetry related ordered stuctures and to maintain ergodicity each was used as a start point for a simulation. $I(\boldsymbol{Q})$ was calcuated at $T=0.3 \mathrm{~K}$ from 200 independent spin configurations derived from each starting structure using the formula 17

$$
I(\boldsymbol{Q}) \propto \frac{|f(Q)|^{2}}{N} \sum_{(i, a),(j, b)} \mathrm{e}^{\imath \boldsymbol{Q} \cdot \boldsymbol{R}_{i j}^{a b}}\left\langle\boldsymbol{S}_{i, \perp}^{a} \cdot \boldsymbol{S}_{j, \perp}^{b}\right\rangle,
$$

where $\langle\ldots\rangle$ denotes a thermal average, $\boldsymbol{S}_{i, \perp}^{a}$ is the spin component perpendicular to the scattering wave vector $\boldsymbol{Q}$ and $f(Q)$ is the magnetic form factor for $\mathrm{Dy}^{3+}$. The Monte Carlo results for $I(\boldsymbol{Q})$ are compared to the experimental data in the $h, h, l$ and $h, k, 0$ planes below. The simulations are nominally at $0.3 \mathrm{~K}$, whereas the experimental data was collected at either $0.3 \mathrm{~K}(h, h, l$ plane) or $0.05 \mathrm{~K}$ ( $h, k, 0$ plane). We consider it valid to make these comparisons because at temperatures below $0.4 \mathrm{~K}$ the susceptibility ${ }^{27}$, neutron scattering intensity, $I(\boldsymbol{Q})$ and specific heat ${ }^{4}$ do not change significantly with temperature: the system essentially appears to be frozen with negligible thermal spin fluctuations.

\section{RESULTS}

In this section, the observed magnetic diffuse scattering is presented and compared to Monte Carlo simulations where possible. The temperature dependence of the scattering in the $h, h, l$ plane is discussed first (section $\amalg$ IIA, in order to establish the temperature regimes of interest. In the following sections (IIIB and IIIC) the scattering intensity measured at base temperature is compared to Monte Carlo simulations. Finally, section IIID is concerned with features in the experimental scattering which are not reproduced by the model. 


\section{A. Temperature Dependence of the Diffuse Scattering}

The temperature dependence of the diffuse scattering due to the development of ice rule fulfilling spin correlations was sampled at four temperatures for the $h, h, l$ plane (20, 1.3, 0.3 and 0.05 K, see Fig. 2). An important point that can be obtained from these measurements is that the diffuse scattering does evolve with temperature. As the sample is cooled, no diffuse scattering characteristic of spin ice correlations has built up by $20 \mathrm{~K}$. This is entirely expected as the bulk susceptibility shows that $\mathrm{Dy}_{2} \mathrm{Ti}_{2} \mathrm{O}_{7}$ is paramagnetic at this temperature 27 . At 1.3 $\mathrm{K}$ strong diffuse scattering has appeared. The form is closely similar to that of the near-neighbor spin ice as simulated in Ref 17 . Between 1.3 and $0.3 \mathrm{~K}$ the spin correlations evolve and additional features appear in the scattering pattern, as discussed below. The scattering at $0.05 \mathrm{~K}$ throughout the $h, h, l$ plane (not illustrated here) is not significantly different to that at $0.3 \mathrm{~K}$.

\section{B. Scattering in the $h, k, 0$ plane}

For the $h, k, 0$ plane, data has only been collected at $0.05 \mathrm{~K}$. The simulation and the experiment seem to agree very well indeed. The two scattering maps are shown in Fig. 3. A comparison by a cut through the data is shown in Fig. 4. The Monte Carlo pattern has been fitted to the experimental diffuse scattering using a flat background and an overall intensity scale parameter.

\section{Scattering in the $h, h, l$ plane}

For the $h, h, l$ plane direct comparison of the experimental and simulated scattering maps reveals both close similarities and significant differences. The two scattering patterns are shown in Fig. 2] It is apparent that whilst the experimental pattern does contain the features of the dipolar spin ice scattering (for example the large diffuse feature at $0,0,3)$ it also contains features that are are not reproduced by the simulation. These extra features are concentrated around the boundaries of the Brillouin zones (the position of the zone boundaries are illustrated in Fig. 2).

The similarities and differences of the observed and calculated patterns are more clearly emphasized in Fig. 4 Here slices through the data have been made. The agreement along $h, h, 0$ and $0,0, l$ is good. The slice along $h, h, 2.3$ is chosen to highlight the differences and it can be seen that where the slice crosses the zone boundary the form of the calculated and experimental intensities is significantly different.

\section{Zone Boundary Features}

One possible explanation for the zone boundary features is that they are due to phonons. However, the following facts suggest that the intensity is magnetic: (1) the scattering appears as temperature is decreased; the converse would be expected for a phonon; (2) the experimentally observed features fall off with increasing $|\mathbf{Q}|$, which is not expected for phonons.

The second point can be demonstrated by comparison of the intensity of the zone boundary features with the magnetic form factor of $\mathrm{Dy}^{3+}$. If the intensity at equivalent points on successive zone boundaries is compared it would be expected to follow the form factor if the scattering process is magnetic. This is found to be the case. On the basis of this comparison it seems entirely reasonable to attribute the zone boundary features to a magnetic correlation.

\section{DISCUSSION}

The dipolar spin ice model apparently provides a very successful model for the zero field spin correlations in $\mathrm{Dy}_{2} \mathrm{Ti}_{2} \mathrm{O}_{7}$. However, some additional spin correlations do occur which are not captured by the model. The model is very successful in describing the bulk and microscopic data for $\mathrm{Ho}_{2} \mathrm{Ti}_{2} \mathrm{O}_{7} \frac{17}{17}$, and the bulk data for $\mathrm{Dy}_{2} \mathrm{Ti}_{2} \mathrm{O}_{7}^{20}$. One might therefore ask, how this further restriction of the dipolar spin ice manifold is not manifested in the fit to the heat capacity?

In answer to this question, it should be noted that the fit to the heat capacity ${ }^{20}$ is not perfect: the experiment and simulation differ in detail. The fact that the zone boundary features are very diffuse in reciprocal space and run all around the zone boundaries, suggests that the associated spin correlations are short ranged and isotropic. A small difference between the experimental and theoretical heat capacities is therefore understandable since the extra spin correlations need not make a major contribution to the entropy. We therefore conclude that the neutron scattering and specific heat data ${ }^{4.20}$ are consistent.

A clue to the origin of the extra spin correlations is the fact that they are much less evident in $\mathrm{Ho}_{2} \mathrm{Ti}_{2} \mathrm{O}_{7}$ 17.18, even if they cannot be excluded completely within experimental error. According to the model, the principal difference between $\mathrm{Ho}_{2} \mathrm{Ti}_{2} \mathrm{O}_{7}$ and $\mathrm{Dy}_{2} \mathrm{Ti}_{2} \mathrm{O}_{7}$ is the more significant antiferromagnetic exchange term in the spin Hamiltonian of $\mathrm{Dy}_{2} \mathrm{Ti}_{2} \mathrm{O}_{7}\left(J=-3.72 \mathrm{~K}\right.$ for $\mathrm{Dy}_{2} \mathrm{Ti}_{2} \mathrm{O}_{7}$ compared to $J=-1.56 \mathrm{~K}$ for $\left.\mathrm{Ho}_{2} \mathrm{Ti}_{2} \mathrm{O}_{7}\right)$. This exchange term was invoked to fit bulk measurements 20.27 .31 , which are not sensitive to the details of a Hamiltonian in the way that diffuse neutron scattering is. Hence the extra scattering might reveal that extra terms are required in the model Hamiltonian: for example, it might be necessary to include further neighbor exchange interactions. 
FIG. 2: (Color online) $\mathrm{Dy}_{2} \mathrm{Ti}_{2} \mathrm{O}_{7}$ : Diffuse scattering in the $h, h, l$ plane measured at $20 \mathrm{~K}$ (top left), $1.3 \mathrm{~K}$ (top right) and 0.3 $\mathrm{K}$ (bottom left, with zone boundaries illustrated). The sharp, intense spots are nuclear Bragg reflections with no magnetic intensity. The map at $20 \mathrm{~K}$ was recorded using an average scattering angle of $\phi=48^{\circ}$ which gives stronger arc-shaped artefacts for small $|\mathbf{Q}|$ values compared to $\phi=32^{\circ}$. The result of the Monte Carlo simulation of the dipolar spin ice model at $0.3 \mathrm{~K}$ is shown at bottom right.

FIG. 3: (Color online) $\mathrm{Dy}_{2} \mathrm{Ti}_{2} \mathrm{O}_{7}$ at $0.05 \mathrm{~K}$ : experimental scattering intensity in the $h, k, 0$ plane (left), compared with that calculated from Monte Carlo simulations (right).

Further theoretical work is required to distinguish various possibilities.

Zone boundary scattering has recently also been observed in the $h, k, 0$ plane for $\mathrm{ZnCr}_{2} \mathrm{O}_{4}{ }^{37}$ and the $h, h, l$ plane for $\mathrm{ZnFe}_{2} \mathrm{O}_{4}{ }^{38}$ (both frustrated antiferromagnets with the spinel structure). We suggest that, while the three magnets are quite different, the zone boundary features may also be a generic feature of frustrated magnets due to the formation of local spin clusters, for example those developing in $\mathrm{ZnCr}_{2} \mathrm{O}_{4}{ }^{37.39}$ which might be at the origin of the "quantum protectorate" state invoked in that system. As noted by Kamazawa et $a l .^{38}$, zone boundary scattering implies correlations between the spins related by fcc translations (as illustrated in Fig. 1). These spins are third neighbors and interactions at this distance will clearly be important in stabilizing a hexagonal cluster. Simulations suggest that other clusters can also be stabilized depending on the details of the Hamiltonian 40 .

In conclusion, we have presented a systematic investigation of the diffuse scattering of $\mathrm{Dy}_{2} \mathrm{Ti}_{2} \mathrm{O}_{7}$ in zero magnetic field. We have observed three regimes of behavior. At $20 \mathrm{~K}$ the spins are paramagnetic, without spin ice correlations, which should help interpret recent bulk susceptibility measurements of the dynamical prop$\operatorname{erties}^{12,13,27}$, which have been discussed in terms of both correlated and uncorrelated dynamical processes 13.15 .16 . At $1.3 \mathrm{~K}$ the spin correlations are similar to those of the near neighbor spin ice model, suggesting that further neighbor dipole interactions are not strongly relevant in this temperature range. At $0.3 \mathrm{~K}$ the scattering is modified to correspond quite closely to the frozen state of the dipolar spin ice model, with effective infinite range interactions. However, in this temperature range we have observed additional scattering features around the zone boundary which for the time being remain unexplained. Further calculations are in progress to clarify their origin.

\section{ACKNOWLEDGEMENTS}

We thank the sample environment team at ISIS, in particular Richard Down and Dave Bates, and Martyn Bull for assistance on PRISMA. TF acknowledges the EPSRC for funding, and Andrew Harrison for hospitality in Edinburgh whilst this work was written up. Work done at the University of Warwick was funded by an EPSRC grant. Work done at the University of Waterloo has been supported by the NSERC of Canada, the Canada Research Chair Program, the Province of Ontario, and Research Corporation.
* Now at Department of Physics, University of Warwick, Coventry, CV4 7AL, United Kingdom

† Now at CEA Grenoble, DRFMC/SPSMS, 38054, Grenoble, Cedex 9, France

¥ Electronic address: s.t.bramwell@ucl.ac.uk

$\S$ Now at Department of Physics, Southern Connecticut State University, New Haven, CT 06515, USA

- Also at Canadian Institute for Advanced Research, 180 Dundas Street West, Toronto, Ontario, M5G 1Z8, Canada

1 M. J. Harris, S. T. Bramwell, D. F. McMorrow, T. Zeiske, and K. W. Godfrey, Phys. Rev. Lett. 79, 2554 (1997).

2 L. Pauling, J. Am. Chem. Soc. 57, 2680 (1935).

3 W. F. Giauque and J. W. Stout, J. Am. Chem. Soc. 58, 1144 (1936).

4 A. P. Ramirez, A. Hayashi, R. J. Cava, R. Siddharthan, and B. S. Shastry, Nature 399, 333 (1999).

5 S. T. Bramwell and M. J. P. Gingras, Science 294, 1495 (2001).

${ }^{6}$ H. Kadowaki, Y. Ishii, K. Matsuhira, and Y. Hinatsu,
Phys. Rev. B 65, 144421 (2002).

7 S. T. Bramwell and M. J. Harris, J. Phys.: Condens. Mat. 10, L215 (1998).

8 M. J. Harris, S. T. Bramwell, P. C. W. Holdsworth, and J. D. M. Champion, Phys. Rev. Lett. 81, 4496 (1998).

9 K. Matsuhira, Z. Hiroi, T. Tayama, S. Takagi, and T. Sakakibara, J. Phys.: Condens. Mat. 14, L559 (2002).

10 T. Sakakibara, T. Tayama, Z. Hiroi, K. Matsuhira, and S. Takagi, Phys. Rev. Lett. 90, 207205 (2003).

11 R. Moessner and S. L. Sondhi, Phys. Rev. B 68, 064411 (2003).

12 K. Matsuhira, Y. Hinatsu, and T. Sakakibara, J. Phys.: Condens. Mat. 13, L737 (2001).

13 J. Snyder, S. Slusky, R. J. Cava, and P. Schiffer, Nature 413, 48 (2001).

14 J. Snyder, J. S. Slusky, R. J. Cava, and P. Schiffer, Phys. Rev. B 66, 064432 (2002).

15 G. Ehlers, A. L. Cornelius, M. Orendáč, M. Kajčnacová, T. Fennell, S. T. Bramwell, and J. S. Gardner, J. Phys.: 
FIG. 4: $\mathrm{Dy}_{2} \mathrm{Ti}_{2} \mathrm{O}_{7}$ at $0.3 \mathrm{~K}$ : comparison of experimental and Monte Carlo data for $h, k, 0$ and $h, h, l$ planes. The comparison in the $h, k, 0$ plane is shown at top left using a slice along $3, k, 0$. The Monte Carlo data was compared to the experimental data by adjusting an overall intensity scale parameter and single background parameter in order to give the best fit. The comparisons for the $h, h, l$ plane are slices along $h, h, 0$ (top right), 0,0,l (bottom left) and along $h, h, 2.3$ (bottom right). For slices 2 and 3 the Monte Carlo data were compared using an overall intensity scale parameter and sloping background, slice 4 was compared with an overall intensity scale parameter and constant background. The slice positions in the scattering plane are indicated in the insets, and for the $h, h, l$ plane by numbers $2-4$. Shaded areas in the slices are Bragg peaks from the sample and were excluded from the fitting.

Condens. Mat. 15, L9 (2003).

16 J. Snyder, B. G. Ueland, J. S. Slusky, H. Karunadasa, R. J. Cava, A. Mizel, and P. Schiffer, Phys. Rev. Lett. 91, 107201 (2003).

17 S. T. Bramwell, M. J. Harris, B. C. den Hertog, M. J. P. Gingras, J. S. Gardner, D. F. McMorrow, A. R. Wildes, A. L. Cornelius, J. D. M. Champion, R. G. Melko, et al., Phys. Rev. Lett. 87, 047205 (2001).

18 M. Kanada, Y. Yasui, Y. Kondo, S. Iikubo, H. Harashina, M. Sato, H. Okumura, K. Kakurai, and H. Kadowaki, J. Phys. Soc. Jpn. 71, 313 (2002).

19 A. preliminary communication of this work appeared in: T. Fennell, O. A. Petrenko, G. Balakrishnan, S. T. Bramwell, J. D. Champion, B. Fåk, M. J. Harris, and D. McK. Paul, Appl. Phys. A 74, S889 (2002).

20 B. C. den Hertog and M. J. P. Gingras, Phys. Rev. Lett. 84, 3430 (2000).

21 R. G. Melko, B. C. den Hertog, and M. J. P. Gingras, Phys. Rev. Lett. 87, 067203 (2001).

22 M. Enjalran and M. J. P. Gingras, arXiv:condmat/0307151 (2003).

23 S. Rosenkranz, A. P. Ramirez, A. Hayashi, R. J. Cava, R. Siddharthan, and B. S. Shastry, J. Appl. Phys. 68, 855 (1992).

24 J. D. Bernal and R. H. Fowler, J. Chem. Phys. 1, 515 (1933).

25 A. L. Cornelius and J. S. Gardner, Phys. Rev. B 64, 060406(R) (2001).

26 O. A. Petrenko, M. Lees, and G. Balakrishnan, Phys. Rev. B 68, 012406 (2003).

27 H. Fukazawa, R. G. Melko, R. Higashinaka, Y. Maeno, and M. J. P. Gingras, Phys. Rev. B 65, 054410 (2002).

${ }^{28}$ R. Higashinaka, H. Fukazawa, and Y. Maeno, Phys. Rev.
B 68, 014415 (2003).

29 B. C. den Hertog, M. J. P. Gingras, S. T. Bramwell, and M. J. Harris, arXiv:cond-mat/9912220 (1999).

${ }^{30}$ Y. Qiu, PhD. Thesis (Johns Hopkins University, Baltimore, 2002).

31 R. Siddharthan, B. S. Shastry, A. P. Ramirez, A. Hayashi, R. J. Cava, and S. Rosenkranz, Phys. Rev. Lett. 83, 1854 (1999).

32 A. Munter, Neutron scattering lengths and cross sections, (www.ncnr.nist.gov).

33 M. F. Collins, Magnetic Critical Scattering (Oxford University Press, Oxford, 1989).

34 G. Balakrishnan, O. A. Petrenko, M. R. Lees, and D. M. Paul, J. Phys.: Condens. Mat 10, L723 (1998).

35 V. F. Sears, J. Appl. Cryst. 17, 226 (1984).

36 R. Melko, M. Enjalran, B. den Hertog, and M. Gingras, arXiv:cond-mat/0308282 (2003).

37 S.-H.Lee, C. L. Broholm, W. Ratcliff, G. Gasparovic, Q. Huang, T. H. Kim, and S.-W. Cheong, Nature 418, 856 (2002).

38 K. Kamazawa, Y. Tsunoda, H. Kadowaki, and K. Kohn, Phys. Rev. B 68, 024412 (2003).

$39 \mathrm{In} \mathrm{ZnCr}_{2} \mathrm{O}_{4}{ }^{37}$ hexagonal loops of spins organize into weakly interacting antiferromagnetic clusters. The fluctuation of the directors of the hexagons governs the low temperature dynamics. This state is termed a "protectorate" as it appears to be a stable state of matter in a strongly correlated system ${ }^{41}$.

40 T. Fennell, T. Yavors'kii, M. J. P. Gingras, J. P. C. Ruff, and M. Weigel, unpublished data.

41 R. B. Laughlin and D. Pines, Proc. Natl. Acad. Sci. USA 97, 28 (2000). 
This figure "fig1a.png" is available in "png" format from: http://arxiv.org/ps/cond-mat/0404594v2 
This figure "fig1b.png" is available in "png" format from: http://arxiv.org/ps/cond-mat/0404594v2 
This figure "fig2a.png" is available in "png" format from: http://arxiv.org/ps/cond-mat/0404594v2 
This figure "fig2b.png" is available in "png" format from: http://arxiv.org/ps/cond-mat/0404594v2 
This figure "fig2c.png" is available in "png" format from: http://arxiv.org/ps/cond-mat/0404594v2 
This figure "fig2d.png" is available in "png" format from: http://arxiv.org/ps/cond-mat/0404594v2 
This figure "fig3a.png" is available in "png" format from: http://arxiv.org/ps/cond-mat/0404594v2 
This figure "fig3b.png" is available in "png" format from: http://arxiv.org/ps/cond-mat/0404594v2 
This figure "fig4a.png" is available in "png" format from: http://arxiv.org/ps/cond-mat/0404594v2 
This figure "fig4b.png" is available in "png" format from: http://arxiv.org/ps/cond-mat/0404594v2 
This figure "fig4c.png" is available in "png" format from: http://arxiv.org/ps/cond-mat/0404594v2 
This figure "fig4d.png" is available in "png" format from: http://arxiv.org/ps/cond-mat/0404594v2 